\title{
Mortality in cancer patients previously diagnosed with herpes zoster in the hospital setting: a nationwide cohort study
}

\author{
S A J Schmidt ${ }^{*}, 1$, G V Sørensen ${ }^{1}$, E Horváth-Puhó ${ }^{1}$, L Pedersen ${ }^{1}$, N Obel ${ }^{2}, \mathrm{~K} \mathrm{~L} \mathrm{Petersen}^{3}$, H C Schønheyder ${ }^{1,4}$ \\ and $\mathrm{H}$ T Sørensen ${ }^{1}$ \\ ${ }^{1}$ Department of Clinical Epidemiology, Aarhus University Hospital, Olof Palmes Allé 43-45, Aarhus N 8200, Denmark; ${ }^{2}$ Department \\ of Infectious Diseases, Copenhagen University Hospital, Rigshospitalet, Blegdamsvej 9, Copenhagen $\varnothing$ 2100, Denmark; \\ ${ }^{3}$ California Pacific Medical Center Research Institute, 475 Brannan Street, Suite 220, San Francisco, CA 94107, USA and \\ ${ }^{4}$ Department of Clinical Microbiology, Aalborg University Hospital, Mølleparkvej 10, Aalborg 9000, Denmark
}

Background: Herpes zoster $(\mathrm{HZ})$ is associated with underlying immunodeficiency and may thereby predict mortality of subsequent cancer.

Methods: By using Danish nationwide medical databases, we identified all cancer patients with a prior hospital-based HZ diagnosis during 1982-2011 ( $n=2754)$ and a matched cancer cohort without prior HZ ( $n=26243)$. We computed adjusted mortality rate ratios (aMRRs) associating prior $\mathrm{HZ}$ with mortality following cancer.

Results: Prior $\mathrm{HZ}$ was associated with decreased mortality within the year after cancer diagnosis (aMRR 0.87; 95\% confidence interval (Cl): 0.81-0.93), but not thereafter (aMRR 1.07; 95\% Cl: 0.99-1.15). However, prior HZ predicted increased mortality throughout the entire follow-up among patients aged $<60$ years (aMRR 1.39; 95\% Cl: 1.15-1.68) and those with disseminated $\mathrm{HZ}$ (aMRR 1.18; 95\% Cl: 1.01-1.37). The increased mortality rates were observed primarily for haematological and immune-related cancers.

Conclusions: Overall, $\mathrm{HZ}$ was not a predictor of increased mortality following subsequent cancer.

Herpes zoster (HZ) may be a marker of occult cancer (Sørensen et al., 2004; Ho et al., 2011; Liu et al., 2012; Wang et al., 2012; Chiu et al., 2013; Cotton et al., 2013; Iglar et al., 2013) and some authors have advocated increased work-up for cancer in patients with $\mathrm{HZ}$ (Zaha et al., 1993; Yamamoto et al., 2003; Cotton et al., 2013). However, limited data are available on whether HZ may be used to predict mortality in patients presenting with subsequent cancer in clinical practice. The two previous studies on the topic overall showed no association (Sørensen et al., 2004; Cotton et al., 2013), but were limited by small sample sizes and lacked information on key prognostic factors, such as cancer stage and comorbidities. The first study, which was conducted by our research group, was furthermore restricted to in-patient HZ diagnoses (Sørensen et al., 2004).
Our objective was to provide updated and more detailed analyses of the association between $\mathrm{HZ}$ and mortality following a subsequent cancer by extending the period of follow-up, by including inpatient, outpatient and emergency room $\mathrm{HZ}$ diagnoses, and by obtaining information on cancer stage, comorbidities and use of immunosuppressive drugs.

\section{MATERIALS AND METHODS}

We conducted this nationwide matched cohort study by using Danish nationwide medical databases. The source population comprised 829787 cancer patients. A detailed description of data

*Correspondence: Dr SAJ Schmidt; E-mail: saj@clin.au.dk

Received 12 December 2014; revised 9 March 2015; accepted 17 March 2015; published online 16 April 2015

(c) 2015 Cancer Research UK. All rights reserved 0007-0920/15 
sources and variable definitions is provided in the Supplementary Table S1.

We identified all patients with a diagnosis of first-time cancer in the Danish Cancer Registry (Statens Serum Institut, 2012) between 1982 and 2011, who had a prior hospital-based (i.e., inpatient, outpatient (ambulatory), or emergency room) HZ diagnosis recorded in the Danish National Patient Registry (DNPR) (Lynge et al., 2011). We considered all available primary and secondary registry diagnoses and used the date of discharge or end of outpatient follow-up as the diagnosis date for HZ. By grouping the diagnosis codes, we differentiated between severity (uncomplicated or complicated) and extent (localised or disseminated) of HZ. For each $\mathrm{HZ}$ patient with subsequent cancer, we sampled up to 10 cancer patients with no prior HZ diagnosis individually matched by sex, birth year, cancer type and calendar period of cancer diagnosis (5-year interval).

From the Danish National Health Service Prescription Database (Johannesdottir et al., 2012) and the DNPR, we retrieved information on the following covariates recorded before cancer diagnosis: treatment with immunosuppressive drugs within the first 6 months, comorbidities associated with immune dysregulation and comorbidity level categorised as none (0), moderate (1), severe $(2)$ or very severe $(\geqslant 3)$ using the Charlson Comorbidity Index score (Charlson et al., 1987).

By using the Civil Registration System (Schmidt et al., 2014), we followed patients from the date of cancer diagnosis until death, emigration, or 31 December 2012, whichever came first. We computed the mortality rate per 1000 person-years for cancer patients with prior $\mathrm{HZ}$ and for the matched cancer cohort. Taking the matching variables into account, we used stratified Cox regression (Hosmer et al., 2008) to compute unadjusted mortality rate ratios (MRRs) with 95\% confidence intervals (CIs) associating prior HZ with mortality following subsequent cancer. Then, we adjusted for comorbidity level and cancer stage (localised, regional, distant or unknown/missing). Because plots of the $\log (-\log$ (survival)) vs log of survival time indicated non-proportionality, we divided follow-up time into $0-1$ year and $>1$ year. We performed analyses for all cancers combined, cancer subgroups (haematological cancers, immune-related cancers, smoking- and alcoholrelated cancers and cancers at all other sites), and individual cancers (Boyle, 1997; Nasca, 2001; Boffetta and Hashibe, 2006). Finally, we examined if the association depended on the included covariates through stratified analyses. As this analysis required dissolving of the matching, we used conventional Cox regression with additional adjustments for matching variables, cancer stage and comorbidity level in each subgroup analysis. All analyses were pre-specified.

\section{RESULTS}

We identified 2795 cancer patients with a prior HZ diagnosis and 26243 cancer comparison cohort members without this diagnosis. Of these, $41(1.5 \%)$ cancer patients with prior HZ were excluded because no matching cancer patients could be identified.

Cancer patients with prior HZ had a median age of 78 years and $56.9 \%$ were women (Table 1). Compared with the matched cancer cohort, HZ patients had a higher prevalence of comorbidities and were more frequently users of immunosuppressive drugs before cancer diagnosis. Cancer stage at time of diagnosis was similar. The observed differences remained when cancer subtypes were examined separately (Supplementary Table S2).

The unadjusted MRR within the first year after cancer diagnosis was 0.95 (95\% CI: 0.89-1.02), but decreased to 0.87 (95\% CI: $0.81-$ 0.93 ) in the full model, which was mainly explained by adjustment for comorbidity level (Table 2). However, the stratified analysis
Table 1. Selected characteristics of persons diagnosed with herpes zoster and subsequent cancer and a matched cancer cohort, Denmark 1982-2011

All malignant neoplasms

\begin{tabular}{|l|c|c|}
\cline { 2 - 3 } & $\begin{array}{c}\text { Herpes zoster } \\
\text { patients }\end{array}$ & $\begin{array}{c}\text { Matched } \\
\text { cohort }\end{array}$ \\
\hline & $\boldsymbol{n}(\%)$ & $\boldsymbol{n}(\%)$ \\
\hline Total & $2754(100.0)$ & $26243(100.0)$ \\
\hline
\end{tabular}

Age at cancer diagnosis (years)

\begin{tabular}{|l|c|c|}
\hline $\begin{array}{l}\text { Range } \\
\text { Median (interquartile range) }\end{array}$ & $\begin{array}{c}18-100 \\
78(70-84)\end{array}$ & $\begin{array}{c}16-102 \\
77(70-84)\end{array}$ \\
\hline Age groups & \\
\hline $15-59$ & $256(9.3)$ & $2259(8.6)$ \\
$60-69$ & $436(15.8)$ & $4344(16.6)$ \\
$70-79$ & $915(33.2)$ & $9105(34.7)$ \\
$\geqslant 80$ & $1147(41.6)$ & $10535(40.1)$
\end{tabular}

Sex

\begin{tabular}{|l|l|l|}
\hline Women & $1566(56.9)$ & $14897(56.8)$ \\
$1188(43.1)$ & $11346(43.2)$ \\
\hline Calendar period of cancer diagnosis & 110 \\
\hline
\end{tabular}

\begin{tabular}{|c|c|c|}
\hline $1982-1994$ & $1020(37.0)$ & $9738(37.1)$ \\
$1995-2011$ & $1734(63.0)$ & $16505(62.9)$ \\
\hline
\end{tabular}

Cancer stage at diagnosis $^{a}$

\begin{tabular}{l|c|c} 
Localised & $1193(43.3)$ & $11489(43.8)$ \\
Regional & $446(16.2)$ & $4151(15.8)$ \\
Distant & $466(16.9)$ & $4592(17.5)$ \\
Unknown/missing & $649(23.6)$ & $6011(22.9)$
\end{tabular}

Charlson Comorbidity Index level ${ }^{\text {b }}$

\begin{tabular}{|l|c|c|}
\hline None & $1236(44.9)$ & $16999(64.8)$ \\
Moderate & $699(25.4)$ & $5366(20.4)$ \\
Severe & $406(14.7)$ & $2362(9.0)$ \\
Very severe & $413(15.0)$ & $1516(5.8)$ \\
\hline Comorbidities with immune dysregulation, & $581(21.1)$ & $2223(8.5)$ \\
overall & & \\
\hline Any autoimmune disease & $514(18.7)$ & $2123(8.1)$ \\
Solid organ transplantation & $46(1.7)$ & $15(0.1)$ \\
Stem cell or bone marrow transplantation & $1(0.0)$ & $3(0.0)$ \\
Human immunodeficiency virus infection & $30(1.1)$ & $41(0.2)$ \\
Primary immunodeficiency & $8(0.3)$ & $16(0.1)$ \\
Otherc & $15(0.5)$ & $58(0.2)$ \\
\hline Immunosuppressive drugs overall $^{\text {d }}$ & $128(4.6)$ & $760(2.9)$ \\
\hline Systemic glucocorticoids & $116(4.2)$ & $663(2.5)$ \\
TNF-alpha inhibitors & - & $2(0.0)$ \\
Other & $31(1.1)$ & $163(0.6)$ \\
\hline
\end{tabular}

Setting of herpes zoster diagnosis ${ }^{\mathbf{e}}$

\begin{tabular}{|l|c|c|}
\hline Inpatient & $2284(82.9)$ & - \\
Outpatient & $289(10.5)$ & - \\
Emergency room & $181(6.6)$ & - \\
\hline
\end{tabular}

\begin{tabular}{|l|c|c|}
\hline \multicolumn{3}{|l|}{ Severity of herpes zoster } \\
\hline $\begin{array}{l}\text { Complicated } \\
\text { Uncomplicated }\end{array}$ & $712(25.9)$ & - \\
\hline Extent of herpes zoster & - \\
\hline Disseminated & $262(9.1)$ & - \\
Localised & $2492(90.5)$ & - \\
\hline Follow-up (years) & $1.71(0.33-4.96)$ & $1.88(0.30-5.47)$ \\
\hline Median (interquartile range) & $0-30.71$ & $0-30.71$ \\
Range & 9470 & 100830 \\
Total &
\end{tabular}

${ }^{a}$ Stage defined according to summary staging as follows: localised (T1-4, N0, M0; Ann Arbour I); regional (T1-4, N1-3, M0; Ann Arbour Ils, II-Ile); distant (T1-4, N1-3, M1; Ann Arbour IIs, III-IV); and unknown or missing.

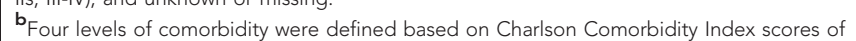
0 (none), 1 (moderate), 2 (severe) and 3 or more (very severe).

'Other immunosuppressive conditions include lymphopenia, leukopenia, agranulocytosis, aplastic anemia and Felty's syndrome.

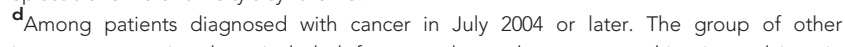
immunosuppressive drugs included, for example, methotrexate, azathioprine, calcineurin inhibitors, other biological agents, and intestinal-acting aminosalicylic acid. See Supplementary Material for a list of included drugs.

eAmong patients diagnosed with zoster in 1995 or later. 
Table 2. Mortality associated with a prior diagnosis of herpes zoster in cancer patients compared with a matched cancer cohort without herpes zoster, Denmark, 1982-2011

\begin{tabular}{|c|c|c|c|c|c|c|c|c|}
\hline & \multicolumn{4}{|c|}{ 0-1 Year of follow-up } & \multicolumn{4}{|c|}{$>1$ Year of follow-up } \\
\hline & \multicolumn{2}{|c|}{$\begin{array}{l}\text { Mortality rate } \\
\text { (per } 1000 \text { PY) }\end{array}$} & \multirow[b]{2}{*}{$\begin{array}{c}\text { Unadjusted } \\
\text { MRR } \\
(95 \% \mathrm{Cl})^{\mathrm{a}}\end{array}$} & \multirow[b]{2}{*}{$\begin{array}{c}\text { Adjusted } \\
\text { MRR } \\
(95 \% \mathrm{Cl})^{b}\end{array}$} & \multicolumn{2}{|c|}{$\begin{array}{l}\text { Mortality rate } \\
\text { (per } 1000 \text { PY) }\end{array}$} & \multirow[b]{2}{*}{$\begin{array}{c}\text { Unadjusted } \\
\text { MRR } \\
(95 \% \mathrm{Cl})^{\mathrm{a}}\end{array}$} & \multirow[b]{2}{*}{$\begin{array}{c}\text { Adjusted } \\
\text { MRR } \\
(95 \% \mathrm{Cl})^{b}\end{array}$} \\
\hline & $\begin{array}{l}\text { Herpes } \\
\text { zoster }\end{array}$ & $\begin{array}{c}\text { Matched } \\
\text { cohort }\end{array}$ & & & $\begin{array}{l}\text { Herpes } \\
\text { zoster }\end{array}$ & $\begin{array}{l}\text { Matched } \\
\text { cohort }\end{array}$ & & \\
\hline All cancers combined & $535(503-567)$ & 539 (528-549) & $0.95(0.89-1.02)$ & $0.87(0.81-0.93)$ & $153(144-162)$ & $122(120-125)$ & $1.20(1.12-1.29)$ & $1.07(0.99-1.15)$ \\
\hline Haematological cancers & $641(520-761)$ & 639 (599-679) & $0.98(0.80-1.20)$ & $0.88(0.71-1.08)$ & $197(160-234)$ & $144(134-153)$ & $1.27(0.99-1.61)$ & $1.22(0.96-1.57)$ \\
\hline Immune-related cancers & $133(106-161)$ & $109(102-117)$ & $1.15(0.92-1.44)$ & $0.94(0.75-1.19)$ & $114(102-125)$ & $86(83-89)$ & $1.30(1.16-1.47)$ & $1.12(1.00-1.27)$ \\
\hline $\begin{array}{l}\text { Smoking- and alcohol- } \\
\text { related cancers }\end{array}$ & 1007 (925-1088) & $1092(1063-1120)$ & $0.90(0.83-0.99)$ & $0.84(0.77-0.92)$ & $220(196-244)$ & $198(191-206)$ & $1.03(0.89-1.19)$ & $0.94(0.81-1.10)$ \\
\hline All other sites & $511(452-570)$ & $500(481-520)$ & $1.00(0.88-1.13)$ & $0.91(0.79-1.03)$ & $158(141-175)$ & $132(127-136)$ & $1.22(1.06-1.39)$ & $1.05(0.91-1.20)$ \\
\hline \multicolumn{9}{|c|}{ 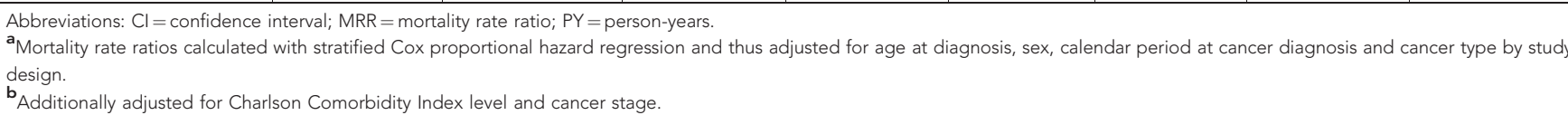 } \\
\hline
\end{tabular}

revealed an increased mortality rate among cancer patients under the age of 60 years (adjusted MRR (aMRR) 1.31; 95\% CI: $0.98-$ 1.74) and among those who had experienced disseminated HZ (aMRR 1.18; 95\% CI: 0.96-1.44) (Table 3). This increase was explained primarily on the basis of the results for haematological cancers (Supplementary Tables S3-S6). The lowest aMRR was observed for patients recently treated with immunosuppressive drugs (aMRR 0.62; 95\% CI: 0.44-0.87 for all cancers combined).

The aMRR was 1.07 (95\% CI: 0.99-1.15) after the first year of follow-up (Table 3). Again, an increased mortality rate was observed primarily for patients under the age of 60 years (aMRR 1.39; 95\% CI: 1.07-1.79) and those with disseminated HZ (aMRR 1.21; 95\% CI: $0.97-1.51$ ) (Table 3). Thus, the youngest patients and patients with prior disseminated $\mathrm{HZ}$ had an increased mortality throughout the entire follow-up period (aMRRs 1.39; 95\% CI: 1.15-1.68 and 1.18; 95\% CI: 1.01-1.37, respectively). The increased estimates were particularly high among the patients with haematological or immune-related cancers (Supplementary Tables S3-S6). The MRRs for individual cancers were imprecise but supported the findings from the main analyses (Supplementary Table S7).

Finally, we observed no substantial difference or specific patterns when stratifying by time between $\mathrm{HZ}$ and cancer diagnoses (Table 3 and Supplementary Tables S3-S6).

\section{DISCUSSION}

We found that a hospital-based $\mathrm{HZ}$ diagnosis predicted an increased mortality in subsequent haematological and immunerelated cancers, particularly among patients aged $<60$ years and among those who had experienced disseminated HZ. For the remaining cancer patients, a prior $\mathrm{HZ}$ diagnosis was associated with a slightly decreased 1-year mortality.

In an earlier study, we found no overall association between HZ and mortality of subsequent cancer except for a potentially increased the mortality (MRR 1.38; 95\% CI: 0.83-2.28) among patients with haematological malignancies diagnosed within a year after HZ (Sørensen et al., 2004). In a British study, median survival was 1197 days among 573 cancer patients ( $87 \%$ of those identified) with prior HZ diagnosed in general practice compared with 1201 days among matched cancer patients without HZ (Cotton et al., 2013). Our data suggest that prior studies may have been confounded by unmeasured comorbidity level. Also, our study allowed us to perform detailed subgroup analyses that revealed potentially important associations.

An intact immune system is essential both for withstanding carcinogenesis (Ershler, 1993; Penn, 2000) and varicella-zoster virus reactivation (Wilson, 2011). HZ could therefore serve as a marker of underlying immune incompetence, possibly explaining the increased mortality of subsequent cancer. However, our results support this hypothesis in only a subgroup of patients. It is possible that disseminated infection and recrudescence in younger patients is a stronger marker of underlying immune incompetence. Indeed, disseminated $\mathrm{HZ}$ occurs more frequently in immunosuppressed individuals and $\mathrm{HZ}$ may be a presenting symptom of human immunodeficiency virus infection (Wilson, 2011; Søgaard et al., 2012). Also, our estimates were driven by haematological and immune-related cancers, which are observed more frequently among immunosuppressed patients (Penn, 2000). Because viruses have been implicated in the development of several of these cancers (Penn, 2000; Alibek et al., 2014), we speculate whether the mechanisms behind immunological evasion of oncogenic viruses and the varicella-zoster virus are closely related. The slightly decreased mortality observed during the first year of follow-up is difficult to reconcile with our a priori hypothesis. Similar stage distribution argues against increased cancer surveillance among $\mathrm{HZ}$ patients as an explanation.

Strength of our study is the nationwide setting in a uniform healthcare system with complete follow-up data (Schmidt et al., 2014). Also, the Danish Cancer Registry has high completeness, maintained by notifications from multiple sources of the healthcare system. High validity (i.e., positive predictive value) is supported by a high proportion of histologically verified tumours (over $90 \%$ for major cancers) and a low proportion ( $0.1 \%)$ of diagnoses based on death certificates only (Statens Serum Institut, 2012).

We believe that the clinical picture of $\mathrm{HZ}$ confers a high diagnostic accuracy (Wilson, 2011) and thus positive predictive value in the DNPR. However, it is possible that physicians underreport $\mathrm{HZ}$ in elderly hospital patients, because they often have other diseases requiring greater clinical attention. Such misclassification could explain the lack of an association among patients aged $\geqslant 60$ years. Unmeasured confounding by lifestyle factors, for example, smoking, could also explain the unexpected results if $\mathrm{HZ}$ is underreported in patients with lifestyle-related comorbidities or if cancer patients with previous $\mathrm{HZ}$ represent healthier persons as they have survived competing diseases after their HZ diagnosis in order to be diagnosed with cancer and thus included in our study. We also lacked data on cancer treatment, 
Table 3. Mortality following cancer among patients with a prior diagnosis of herpes zoster compared with a matched cancer cohort, by study characteristics, Denmark 1982-2011

\section{0-1 Year of follow-up}

$>1$ Year of follow-up

Mortality rate (per 1000 PY)

Mortality rate (per 1000 PY)

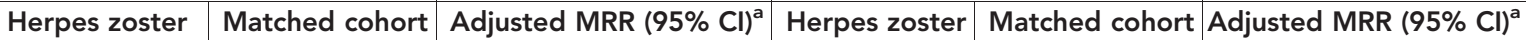

Time between herpes zoster and cancer diagnoses

\begin{tabular}{|c|c|c|c|c|c|c|}
\hline $\begin{array}{l}\text { 0-365 Days } \\
>365 \text { Days }\end{array}$ & $\begin{array}{l}572(481-664) \\
529(495-563)\end{array}$ & $\begin{array}{l}602(572-633) \\
529(518-540)\end{array}$ & $\begin{array}{l}0.86(0.72-1.03) \\
0.87(0.81-0.94)\end{array}$ & $\begin{array}{l}152(128-177) \\
153(144-163)\end{array}$ & $\begin{array}{l}125(119-132) \\
122(119-124)\end{array}$ & $\begin{array}{l}1.13(0.92-1.39) \\
1.06(0.97-1.14)\end{array}$ \\
\hline \multicolumn{7}{|l|}{ Sex } \\
\hline $\begin{array}{l}\text { Women } \\
\text { Men }\end{array}$ & $\begin{array}{l}511(469-552) \\
568(517-618)\end{array}$ & $\begin{array}{l}514(501-528) \\
571(555-588)\end{array}$ & $\begin{array}{l}0.91(0.84-0.99) \\
0.87(0.79-0.96)\end{array}$ & $\begin{array}{l}144(133-154) \\
170(154-185)\end{array}$ & $\begin{array}{l}115(112-118) \\
135(131-139)\end{array}$ & $\begin{array}{l}1.08(0.99-1.17) \\
1.11(1.00-1.22)\end{array}$ \\
\hline
\end{tabular}

Age at cancer diagnosis

\begin{tabular}{|c|c|c|c|c|c|c|}
\hline 15-59 Years & $294(222-366)$ & $192(173-212)$ & $1.31(0.98-1.74)$ & $62(48-75)$ & $36(33-39)$ & 1.39 (1.07-1.79) \\
\hline 60-69 Years & 332 (272-391) & 347 (328-366) & 0.85 (0.71-1.03) & $103(88-118)$ & 78 (74-82) & $1.09(0.93-1.28)$ \\
\hline 70-79 Years & 557 (500-614) & $566(548-584)$ & $0.86(0.77-0.96)$ & 152 (137-167) & $129(124-133)$ & $1.03(0.93-1.14)$ \\
\hline$\geqslant 80$ Years & $680(622-739)$ & 713 (693-733) & $0.88(0.81-0.97)$ & 262 (239-285) & 224 (217-231) & 1.08 (0.98-1.19) \\
\hline \multicolumn{7}{|c|}{ Calendar period of cancer diagnosis } \\
\hline 1982-1994 & 706 (642-769) & $698(678-719)$ & $0.91(0.83-1.00)$ & $160(146-173)$ & $136(132-140)$ & $1.06(0.97-1.16)$ \\
\hline 1995-2011 & 448 (412-484) & $456(445-468)$ & $0.87(0.80-0.95)$ & $148(137-160)$ & 112 (109-115) & $1.12(1.03-1.22)$ \\
\hline
\end{tabular}

\begin{tabular}{|c|c|c|c|c|c|c|}
\hline \multicolumn{7}{|c|}{ Stage at cancer diagnosis } \\
\hline Local & 225 (196-253) & $188(180-196)$ & $0.97(0.84-1.11)$ & 129 (119-139) & $99(96-101)$ & $1.13(1.04-1.23)$ \\
\hline Regional & 582 (498-665) & 657 (628-687) & $0.84(0.72-0.97)$ & 182 (155-208) & 165 (157-174) & $1.03(0.89-1.20)$ \\
\hline Metastatic & 1958 (1757-2159) & 1993 (1928-2058) & $0.92(0.82-1.02)$ & $421(330-511)$ & 365 (340-390) & $1.02(0.81-1.28)$ \\
\hline Unknown/missing & 635 (561-709) & 694 (668-719) & $0.85(0.75-0.96)$ & 185 (161-209) & 150 (143-157) & $1.09(0.95-1.25)$ \\
\hline \multicolumn{7}{|c|}{ Charlson Comorbidity Index level } \\
\hline None & $422(381-463)$ & 447 (436-459) & $0.92(0.83-1.02)$ & $126(115-136)$ & 105 (103-108) & $1.10(1.01-1.20)$ \\
\hline Moderate & 604 (536-673) & 659 (632-685) & $0.89(0.79-1.01)$ & 172 (152-193) & 172 (164-179) & $1.06(0.94-1.20)$ \\
\hline Severe & $673(574-771)$ & 771 (726-815) & $0.86(0.74-1.01)$ & 198 (168-229) & $196(183-210)$ & $1.04(0.88-1.23)$ \\
\hline Very severe & 665 (570-760) & 993 (926-1059) & $0.79(0.68-0.93)$ & 225 (189-260) & $260(236-284)$ & $1.03(0.85-1.24)$ \\
\hline \multicolumn{7}{|c|}{ Comorbidity with immune dysregulation } \\
\hline Yes & 517 (449-585) & $576(538-613)$ & $0.85(0.73-0.98)$ & 150 (131-170) & $136(126-146)$ & $1.01(0.87-1.18)$ \\
\hline No & $540(504-576)$ & $535(524-546)$ & $0.90(0.84-0.97)$ & $154(144-164)$ & $121(119-124)$ & $1.11(1.03-1.18)$ \\
\hline \multicolumn{7}{|c|}{ Immunosuppressive drugs ${ }^{b}$} \\
\hline Yes & $427(293-561)$ & 646 (574-719) & $0.62(0.44-0.87)$ & $130(82-179)$ & 149 (124-175) & $0.75(0.49-1.15)$ \\
\hline No & $337(290-385)$ & $336(321-351)$ & $0.87(0.75-1.01)$ & $129(109-149)$ & $107(101-113)$ & $0.98(0.83-1.16)$ \\
\hline \multicolumn{7}{|c|}{ Setting of herpes zoster diagnosis ${ }^{c}$} \\
\hline Inpatient & 469 (405-534) & $421(401-441)$ & $0.98(0.84-1.13)$ & $148(127-170)$ & $110(105-116)$ & $1.09(0.93-1.27)$ \\
\hline Outpatient clinic & $388(304-471)$ & 411 (382-439) & $0.89(0.70-1.11)$ & $124(95-152)$ & $103(95-111)$ & $1.02(0.80-1.31)$ \\
\hline Emergency room & $296(207-384)$ & 345 (314-376) & $0.76(0.56-1.04)$ & 107 (78-136) & 89 (81-98) & $1.05(0.78-1.40)$ \\
\hline \multicolumn{7}{|c|}{ Severity of herpes zoster } \\
\hline Uncomplicated & $531(494-568)$ & 549 (537-562) & $0.86(0.80-0.92)$ & $157(147-168)$ & $123(120-125)$ & $1.10(1.02-1.18)$ \\
\hline Complicated & $545(482-609)$ & $508(488-528)$ & $0.97(0.86-1.10)$ & $141(125-158)$ & $122(117-126)$ & $1.06(0.94-1.21)$ \\
\hline \multicolumn{7}{|c|}{ Extent of herpes zoster } \\
\hline Localised & $531(498-565)$ & $544(533-555)$ & $0.87(0.81-0.93)$ & $153(144-162)$ & $122(120-125)$ & $1.08(1.01-1.15)$ \\
\hline Disseminated & $569(460-677)$ & $486(454-517)$ & $1.18(0.96-1.44)$ & 158 (125-191) & $123(115-132)$ & $1.21(0.97-1.51)$ \\
\hline \multicolumn{7}{|c|}{ 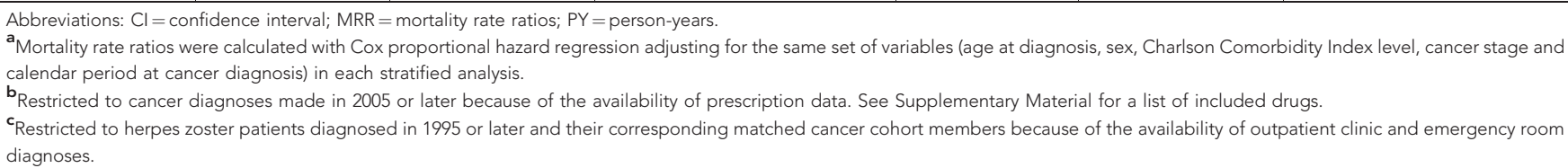 } \\
\hline
\end{tabular}

but because major determinants of treatment choice (i.e., age, stage and comorbidity) were included, we do not believe that it had major impact on our results. However, residual confounding because of missing information on stage is possible, in particular for haematological cancers. Nevertheless, inclusion of this variable in the model did not suggest that it was a major confounder.

Because the observed association depended on HZ severity, our findings may not be applicable to mild HZ treated in general practice. Furthermore, members of the comparison cohort may have had $\mathrm{HZ}$ treated in general practice, possibly resulting in bias towards the null. Misclassification in subgroups of severity and extent of $\mathrm{HZ}$ is another limitation as some ICD-8 codes are unspecific. Because we grouped such codes as uncomplicated and localised, it cannot explain the increased MRRs observed for disseminated $\mathrm{HZ}$.

Finally, we performed several subgroup analyses, which may have resulted in some spurious findings. However, the particularly increased MRRs found for the youngest age group and disseminated $\mathrm{HZ}$ were consistent for both haematological and immunerelated cancers.

In conclusion, hospital-based $\mathrm{HZ}$ did not predict increased mortality of subsequent cancer except in patients with haematological or immune-related cancer, particularly among patients aged $<60$ years and those with disseminated infection. 


\section{ACKNOWLEDGEMENTS}

The study was supported by the Program for Clinical Research Infrastructure (PROCRIN) established by the Lundbeck Foundation and the Novo Nordisk Foundation, as well as by grants from the Danish Cancer Society (R73-A4284-13-S17), from Aarhus University Research Foundation, from Clinical Epidemiological Research Foundation and from Kgl. Hofbuntmager Aage Bangs Fond. The study was approved by the Danish Data Protection Agency (record number 1-16-02-1-08). Danish legislation does not require ethical review board approval or informed consent from subjects for registry-based studies.

\section{CONFLICT OF INTEREST}

The authors declare no conflict of interest.

\section{REFERENCES}

Alibek K, Baiken Y, Kakpenova A, Mussabekova A, Zhussupbekova S, Akan M, Sultankulov B (2014) Implication of human herpesviruses in oncogenesis through immune evasion and supression. Infect Agents Cancer 9: 3.

Boffetta P, Hashibe M (2006) Alcohol and cancer. Lancet Oncol 7: 149-156.

Boyle P (1997) Cancer, cigarette smoking and premature death in Europe: a review including the Recommendations of European Cancer Experts Consensus Meeting, Helsinki, October 1996. Lung Cancer 17: 1-60.

Charlson ME, Pompei P, Ales KL, MacKenzie CR (1987) A new method of classifying prognostic comorbidity in longitudinal studies: development and validation. J Chronic Dis 40: 373-383.

Chiu H-F, Chen BK, Yang C-Y (2013) Herpes zoster and subsequent risk of cancer: a population-based study. J Epidemiol 23: 205.

Cotton SJ, Belcher J, Rose P, K Jagadeesan S, Neal RD (2013) The risk of a subsequent cancer diagnosis after herpes zoster infection: primary care database study. $\mathrm{Br}$ J Cancer 108: 721-726.

Ershler WB (1993) The influence of an aging immune system on cancer incidence and progression. J Gerontol 48: B3-B7.

Ho JD, Xirasagar S, Lin HC (2011) Increased risk of a cancer diagnosis after herpes zoster ophthalmicus: a nationwide population-based study. Ophthalmology 118: 1076-1081.

Hosmer DW, Lemeshow S, May S (2008) Extensions of the proportional hazards model. In Applied Survival Analysis, Badling JD, Cressie NAC,
Fitzmaurice GM, Johnstone IM, Molenberghs G, Scott DW, Smith AFM, Tsay RS, Weisberg S (eds) John Wiley \& Sons, Inc.: Hobroken, New Jersey. Iglar K, Kopp A, Glazier RH (2013) Herpes zoster as a marker of underlying malignancy. Open Med 7: e68-e73.

Johannesdottir SA, Horvath-Puho E, Ehrenstein V, Schmidt M, Pedersen L, Sørensen HT (2012) Existing data sources for clinical epidemiology: The Danish National Database of Reimbursed Prescriptions. Clin Epidemiol 4: 303-313.

Liu Y-C, Yang Y-H, Hsiao H-H, Yang W-C, Liu T-C, Chang C-S, Yang M-Y, Lin P-M, Hsu J-F, Chang P-Y, Lin S-F (2012) Herpes zoster is associated with an increased risk of subsequent lymphoid malignancies-a nationwide population-based matched-control study in Taiwan. BMC Cancer 12: 503.

Lynge E, Sandegaard JL, Rebolj M (2011) The Danish National Patient Register. Scand J Public Health 39: 30-33.

Nasca PC (2001) Immunity and cancer risk. In Fundamentals of Cancer Epidemiology, Nasca PC, Pestides H) (eds) Aspen Publishers, Inc.: Gaithersburg, NY, pp 225-273.

Penn I (2000) Post-transplant malignancy: the role of immunosuppression. Drug Saf 23: 101-113.

Schmidt M, Pedersen L, Sørensen HT (2014) The Danish Civil Registration System as a tool in epidemiology. Eur J Epidemiol 29: 541-549.

Statens Serum Institut (2012) The modernized cancer registry - method and quality (in Danish). December 2009. Available at http://www.ssi.dk/ / media/Indhold/DK\%20-\%20dansk/Sundhedsdata\%20og\%20it/NSF/ Registre/Cancerregisteret/Det $\% 20$ moderniserede $\% 20$ Cancerregister $\% 20 \%$ 20metode\%20og\%20kvalitet.ashx.

Søgaard OS, Lohse N, Østergaard L, Kronborg G, Røge B, Gerstoft J, Sorensen HT, Obel N (2012) Morbidity and risk of subsequent diagnosis of HIV: a population based case control study identifying indicator diseases for HIV infection. PLoS ONE 7: e32538.

Sørensen HT, Olsen JH, Jepsen P, Johnsen SP, Schønheyder HC, Mellemkjaer L (2004) The risk and prognosis of cancer after hospitalisation for herpes zoster: a population-based follow-up study. Br J Cancer 91: 1275-1279.

Wang YP, Liu CJ, Hu YW, Chen TJ, Lin YT, Fung CP (2012) Risk of cancer among patients with herpes zoster infection: a population-based study. CMAJ 184: E804-E809.

Wilson JF (2011) Herpes zoster. Ann Intern Med 154: ITC31-15.

Yamamoto M, Mine H, Akazawa K, Maehara Y, Sugimachi K (2003) Gastrointestinal cancer and herpes zoster in adults. Hepatogastroenterology 50: 1043-1046.

Zaha M, Hayashi I, Odashiro M, Mizoguchi H, Fujiwara M, Kato H, Kawamura J (1993) Herpes zoster and malignancy. Masui 42: 1343-1346.

This work is published under the standard license to publish agreement. After 12 months the work will become freely available and the license terms will switch to a Creative Commons AttributionNonCommercial-Share Alike 4.0 Unported License.

Supplementary Information accompanies this paper on British Journal of Cancer website (http://www.nature.com/bjc) 